\title{
s-ITSF: a service based intelligent transportation system framework for smart accident management
}

\author{
Jeong Kyu Lee ${ }^{1}$, Young Sik Jeong ${ }^{2}$ and Jong Hyuk Park ${ }^{1 *}$
}

\section{${ }^{*}$ Correspondence:} parkjonghyuk1@hotmail.com; jhpark1@seoultech.ac.kr ${ }^{1}$ Department of Computer Science and Engineering, Seoul National University of Science and Technology, Seoul, South Korea Full list of author information is available at the end of the article

\begin{abstract}
Recently, traffic jams and accidents increase due to increase of traffic volume. Thus, intelligent transport system (ITS) is developed to provide efficient road situation and information is actively studying. However, it is mainly focused on traffic jam and management of traffic situation that a systematic management is not provided when an accident occurs. An action to prevent an accident providing the reliable information and a systematic management after accident is needed. In this paper we propose a service based intelligent transportation system framework (s-ITSF) to provide efficient and systematic accident management. The s-ITSF provides an efficient ITS service such as before/after accident management using the accident prevention and management and traffic information data through provision of highly reliable information through encryption and authentication based on vehicular cloud computing (VCC) environment. In addition, it provides systematic accident management which may reduce loss of lives and properties from the traffic accident by quick settlement.
\end{abstract}

Keywords: ITS, VCC, Accident management, Authentication

\section{Background}

Vehicular computing is used to provide traffic jam and efficient road situations according to increase of traffic volume has been actively studying. In addition, VCC engrafts the cloud computing on vehicle computing facilitates multi settlement from the information of single vehicle to the information of many vehicles [1]. However, the traffic management system has been developed due to technical convergence of ITS and VCC but the traffic accident management to provide systematic subsequent action has not been efficiently developed. The traffic accident entails additional damages such as traffic jam, time and economic losses due to the secondary accident. In order to reduce damages and control or handle the situation of the accident promptly, a smart accident management system provides an active and systematic management in addition it helps an accident settlement is needed. Before an accident occurs, smart accident management (SAM) carries the out management of accident prevention, driver and analyzing the vehicle and driver conditions. After an accident occurs, The SAM provides a service in hierarchy according to the level of accident damage and checking the conditions of vehicle and health condition of driver.

(c) 2015 Lee et al. This article is distributed under the terms of the Creative Commons Attribution 4.0 International License (http:// creativecommons.org/licenses/by/4.0/), which permits unrestricted use, distribution, and reproduction in any medium, provided you give appropriate credit to the original author(s) and the source, provide a link to the Creative Commons license, and indicate if changes were made. 
This paper presents a system framework which support and provide a prompt response to the accident by efficiently managing the accident and informing applicable authorities including control center through a systematic accident management system. Safety is available under ITS environment. This paper explains the research trend of key technology and accident management and system requirements in Chapter 2, proposes an accident management system, service scenario and comparison analysis between previous proposes and the propose proposed by this paper in Chapter 3, and finally concludes in Chapter 4.

\section{Related works}

This chapter, discussed about the introduction to mobile ad hoc networks and vehicular ad hoc network. It also discussed about researches like decision support system, connected vehicle technology, vehicular cyber-physical systems and system considerations for accident management.

\section{Vehicular ad hoc network (VANET)}

VANET is one of mobile ad hoc networks (MANET) and ad hoc network formed by vehicles equipped wireless communication technology. In VANET, each vehicle as a communication node and may exchange the information through the communication between vehicles or among road side units (RSUs) [2]. As the vehicles rapidly increase and wireless communication technology develops, VANET related researches have been actively carried out. VANET, a special type of MANET, performs multi-hop communication among nodes without infrastructure which but it accompanied frequent topology variation and network suspension due to high mobility of vehicles. In order to solve the problems, a protocol suitable to the situation based on efficient VANET between vehicles and RSUs will be used to provide more effective accident management service [3]. Each vehicle acts as a transmitter and receiver and delivers the information to near vehicles, competent authority and data center through RSUs when an accident takes place.

Vinh Hoa LA presented a survey of VANETs attacks and solutions in carefully considering other similar works as well as updating new attacks and categorizing them into different classes [4]. And also J. Li put forward a new routing protocol called VDLA, which is aimed at finding an optimal route that can reduce transmission delay and hop count and achieve a high packet delivery rate at the same time. VDLA adopts a junction-based geographic routing approach. VDLA incorporates the traffic density information, the distance to the destination, and the network traffic load when select the next intersection [5]. Finally, Mohamed Hamdi presented the communication architecture of VANETs and outlines the privacy and security challenges that need to be overcome to make such networks safety usable in practice. It identified all existing security problems in VANETs and classifies them from a cryptographic point of view. It regrouped, studied and compared also the various cryptographic schemes that have been separately suggested for VANETs, evaluates the efficiency of proposed solutions and explores some future trends that will shape the research in cryptographic protocols for intelligent transportation systems [6]. 


\section{The existing researches}

Alvert et al. proposed decision support system (DSS) which detects an accident that may occur in the tunnel. This is a system which provides an operator that treats the system in real time based on a certain method through SOS such as automatic incident detection (AID), CCTV or fire detection system when an emergency takes place in the tunnel. It forecasts an urgent situation to occur in the tunnel and provides the information for a fire, number of the wounded, vehicles locked in the tunnel to advise the seriousness of the accident. In addition, when it is necessary, it also provides the information obtained from the accident such as number of lanes of the tunnel, CC cameras, length and width of the tunnel and other provability data including possibility of the injured and serious injured through the system integrating accident model, evacuation model and decision model [7].

$\mathrm{Ni}$ et al. proposed Cyber-Physical Solution which provides distributed processing using connected vehicle technology (CVtech) in order to mitigate the problem of safety and efficiency of the traffic system. Since the current traffic system decides next act and controls the vehicle through monitoring surroundings of the road by driver, it solely relies the path selection on the driver. Most of the accidents take place due to wrong decision of the drivers. Thus, the communication among vehicles $(\mathrm{V}-\mathrm{V})$, Roadside infrastructures (VR), and Personal communication devices (VP) will be used in order to solve such limited problem. They avoid conflicts and jam being connected to external area and environment and captures interaction of the vehicles through Traffic Cyber-Physical System via distributed processing to provide integrated viewpoint in order to warn the accident risk in advance and support the driver to run a vehicle safely. In addition, they integrate the parts information and use the network, embedded sensors, computer database and real time communication which are perfectly suitable to the traffic infrastructure including physical elements such as the status around the road. They also provide the current knowledge based on traffic cyber-physical system with integrated online system modeling and tools by integrating the basic theory of the elements and cyber and physical process [8].

Wan et al. proposed vehicular cyber-physical systems (VCPS) and mobile cloud computing (MCC), integration architecture (VCMIA) to support an intelligent traffic system and cloud service for smooth traffic by integrating interactions between VCPS and MCC. Geographic information is provided through geographic information system (GIS) and the maintenance service of the vehicle is verified by vehicle maintenance service (VMS). VCPS verifies an application for improved traffic safety and driving and provides GIS through integrated recognition mobile. It also shares the entertainment resources and safety information of the driver and passengers through VANET vehicle cluster. It also delivers the accident information to the near drivers promptly when an accident took place. Macro layer controls all types of services through the service center to provide high quality services [9].

\section{System considerations for accident management}

\section{Active process}

Active processing is necessary in order to reduce the damages from the traffic accidents in the road traffic situation. It should have features to prevent an accident actively and 
provide a systematic settlement when an accident takes place. Active process has a close relation between not only physical and economic damages but also lives of the driver, passengers and pedestrians. Therefore, more prompt accident settlement should be provided when an accident takes place [10].

\section{Traffic prevention/management process}

Prevention/management processing is necessary in order to induce a correct judgment of the driver and provide a prompt settlement of an accident on the road traffic situation. The currents model proposes prevention of accident but provide insufficiency in the post-accidents situation. A prompt and systematic settlement may simply prevent an accident and also reduce the secondary and tertiary damages including loss of lives. With above reasons, a more safety accident management service shall be provided in the road traffic situation.

\section{Data authentication}

The reliability of transmitted data in the multi-users environment such as VCC environment is very important. The information of the users, vehicle conditions and user's health are sensitive data. In addition, when the data are forged or falsified, a great amount of manpower and resources shall be wasted and the life of user could be directly affected. Thus, authenticated data must be provided in order to minimize the damages and provide highly reliable data [11].

\section{The proposed s-ITSF}

This paper proposed service based intelligent transportation system framework (s-ITSF) to provide an active and systematic accident management. This chapter consists of s-ITSF architecture, user authentication and service scenario.

s-ITSF prevents an accident and reduces the frequency of accidents through the information of road condition before accident, and provides an accident management in hierarchy by defining the status of accident after accident.

s-ITSF consists of transportation and accident databases center (TADC), accident management center (AMC) and accident management organization (AMO).

TADC checks the accident history of the past, accident occurrence frequency and continuous accident occurrence section by managing and storing the data for vehicles and accidents. Therefore, it defines the dangerous section and systematically manages such sections by providing the information. It checks the condition of driver and vehicle through user health check service, condition check and classification, vehicle state check service to judge whether a safe driving is possible before driving. In addition, it prevents an accident by providing pre-collision service which may prevent the collision between the vehicles under travel and lane keeping service to avoid lane departure. A convenient and safe driving is possible through by providing fuel service and battery/tire exchange service. It classifies the user who is able to drive the vehicle by user authentication in order to receive the health information of the user when an accident occurred and provides a security to the sensitive health information through encryption. It provides all about accident information with AMO in order to offer the accident overview, cause, road condition, countermeasures and subsequent action when an accident occurs. 
AMC classifies the status of the accident through the user's health information and status information vehicle collected in order to classify the exact accident status when an accident occurs. vehicle damage level (VDL) VDL-0 provides a service which checks the normal status vehicle of such as driver's condition, lane keeping, and prevention of collision, fuel status and batter/tire status. VDL-1 provides a service which checks a minor accident of the vehicle such as driver's health condition and vehicle status and authenticates the trueness of the accident through black box service. It also informs the accident to insurance company for quick settlement. VDL-2 and 3 provides a service which checks general accident status and large accident status such as alarm service of airbag and secondary accident warning to inform the accident status to the family of the user. In addition, it provides an efficient service through the ambulance and police car service for safety of the driver. Through the accident management service classified by hierarchy, it may provide a quick and safe service minimizing unnecessary manpower and waste of resources when an accident takes place. Further, it delivers the accident status and position information to TADC based on the information of user's health and vehicle's status collected.

AMO provides a value added service such as insurance and spare parts of the vehicle through Ambulance, Insurance, Vehicle Shop and Tow Service through the accident status information delivered through TADC. In addition, Police Service provides a legal issue settlement after accident by quick accident processing management (Fig. 1).

\section{s-ITSF authentication}

User authentication is essentially needed in order to utilize s-ITSF. The health condition of the user shall be checked and driving the vehicle shall be limited through user authentication. User will be transmit the availability of driving to TADC. In the Fig. 2, it provides the confidentiality through encryption and authentication scheme with an

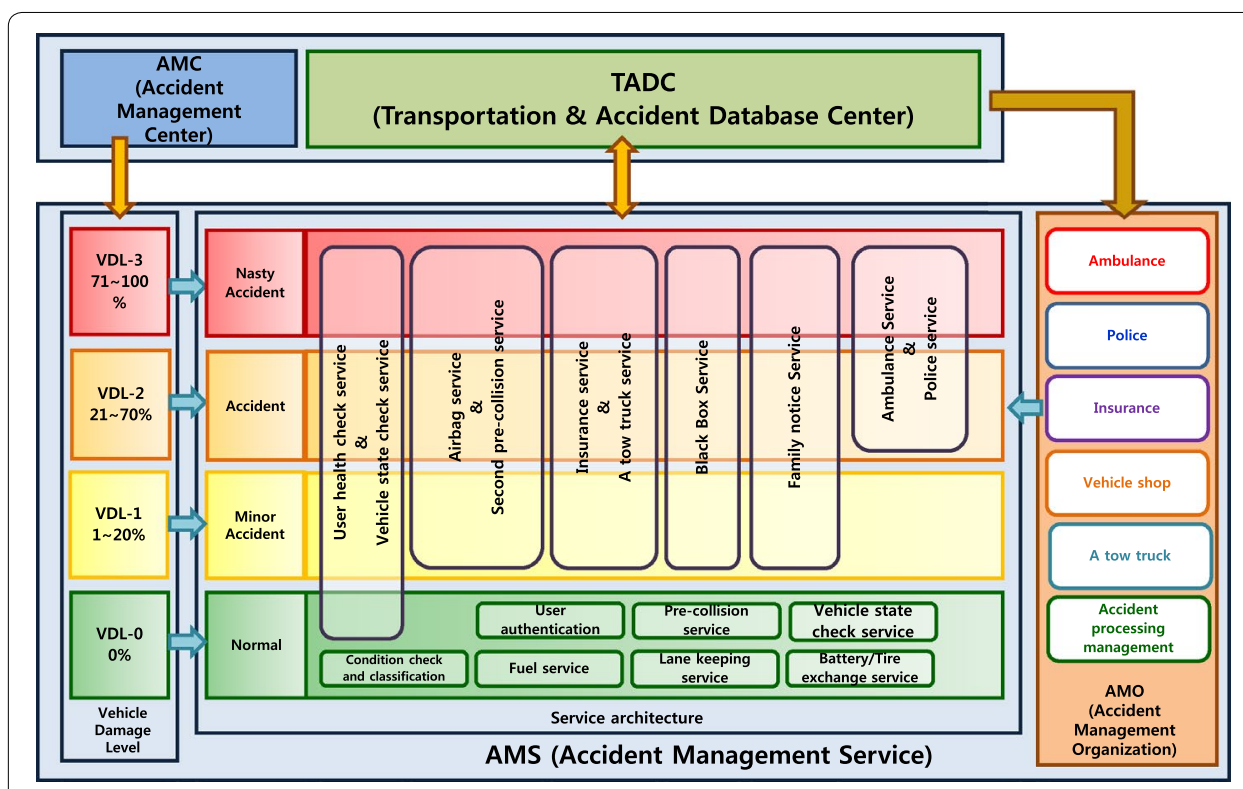

Fig. 1 Framework of s-ITSF 
enhanced security function which will be implemented in Man in the Middle Attack (MITM). Table 1 describes the acronyms which are used in authentication of s-ITSF.

Step 1 User, authentication procedure requests authentication providing vehicle, vehicle with $U H D_{U}$ together with $T_{1}$, a time parameter of user

Step $2 U H D_{U}$, authenticated value and $M_{V}$ which shows a possibility of driving should be encrypted through user's individual key, $P R_{U}$ to authenticate by transmitting E (PRU, $\left.\left[M_{\mathrm{V}}\left\|U H D_{\mathrm{U}}\right\| T_{1}\right]\right)$

Step 3 Transmits the user's conditions and possibility of driving $\mathrm{E}\left(P U_{\mathrm{T}}\right.$, $\left.\left[M_{\mathrm{V}} \| U H D_{\mathrm{U}}\right]\right)$ to TADC encrypting authenticated $U H D_{U}$ and $M_{V}$ to an open key, $P U_{R}$

Step 4 TADC requests VEH to inform $V D_{V E H}$ and $T_{2}$, time parameter for authentication of vehicle after user authentication

Step 5 Authenticates by transmitting the vehicle information, E (PR , $\left[P U_{\mathrm{U}}||\right.$ Request ||$\left.V D_{\mathrm{VEH}} \| T_{2}\right]$ ) including $V D_{\mathrm{VEH}}$ and $T_{2}$, by encrypting to vehicle's individual key, $P R_{U}$

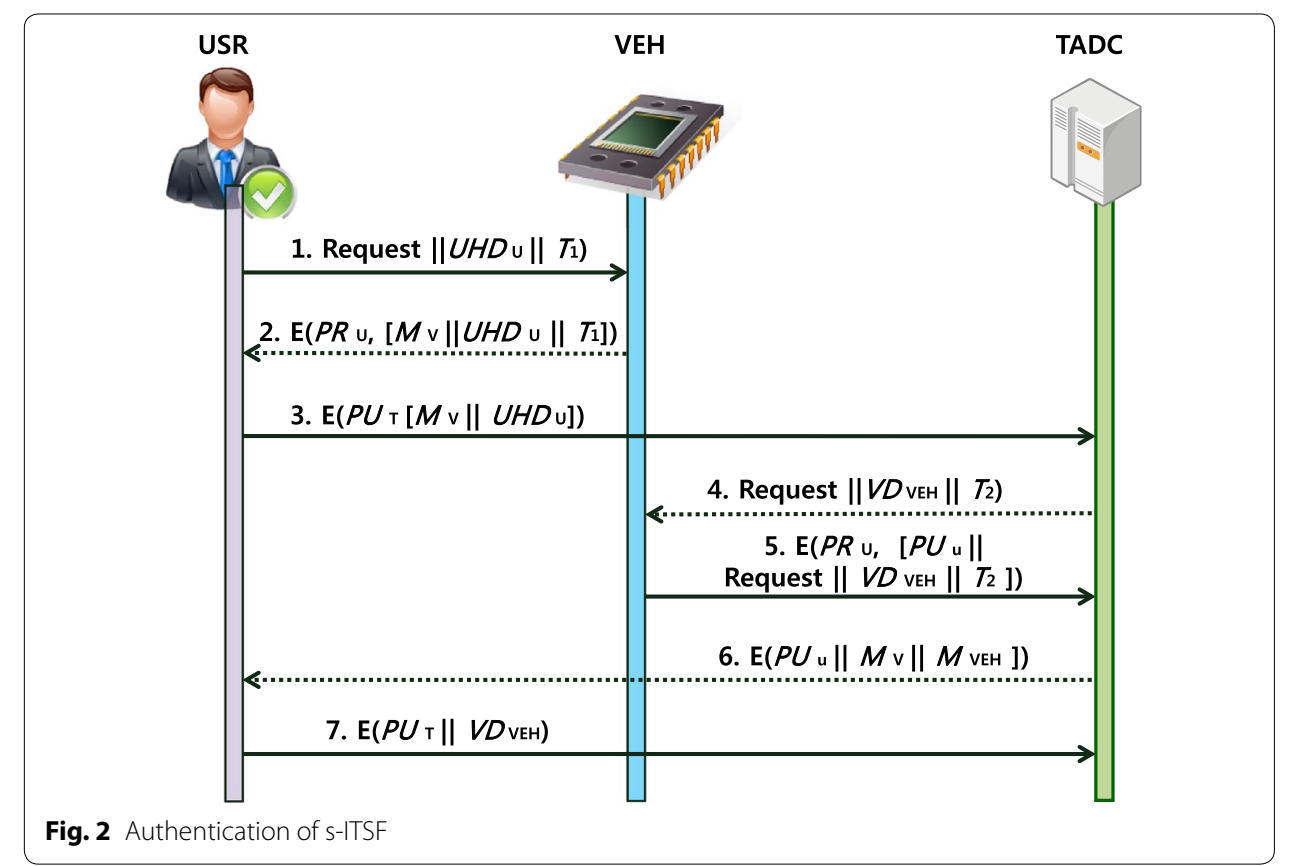

Table 1 Definition of acronyms

\begin{tabular}{ll}
\hline Notation & Description \\
\hline USR & User \\
VEH & Vehicle \\
TADC & Transportation/accident database center \\
PR/PU & Private key/public key \\
UHD/ND & User health data/vehicle data \\
$M_{V}$ & Driving possible/impossible message \\
$M_{\text {VEH }}$ & Vehicle state data \\
\hline
\end{tabular}


Step 6 TADC delivers the encrypted information $\mathrm{E}\left(P U_{\mathrm{U}}\left\|M_{\mathrm{V}}\right\| M_{\mathrm{VEH}}\right)$, Driving possible/impossible information and Vehicle states information to the driver all information encrypted by $P U_{U}$

Step 7 User delivers $\mathrm{E}\left(P U_{\mathrm{U}}|| V_{\mathrm{VEH}}\right)$, the authenticated vehicle information, $V D_{\mathrm{VEH}}$ to TADC

\section{s-ITSF service scenario}

This section proposed s-ITSF for an efficient and systematic accident management. The configuration of s-ITSF, a framework proposed by this paper is shown in Fig. 3 when an accident occurs.

Traffic jam, time and economic loss and additional damage occurs due to traffic accident in ITS environment. This paper has used the method of Fig. 4 in order to solve such problem. When an accident occurs after detecting an accident, it informs the accident status by transmitting the accident information to TADC. AMC provides a service applicable by classifying the accident according to the levels defined through the accident information collected.

\section{Performance analysis of s-ITSF}

This section analyses the system proposed based on major elements discussed in consideration of s-ITSF proposed (Table 2).

s-ITSF provides not only prevention of accident but also a prompt and systematic action through the management after accident. Above all, active processing is needed

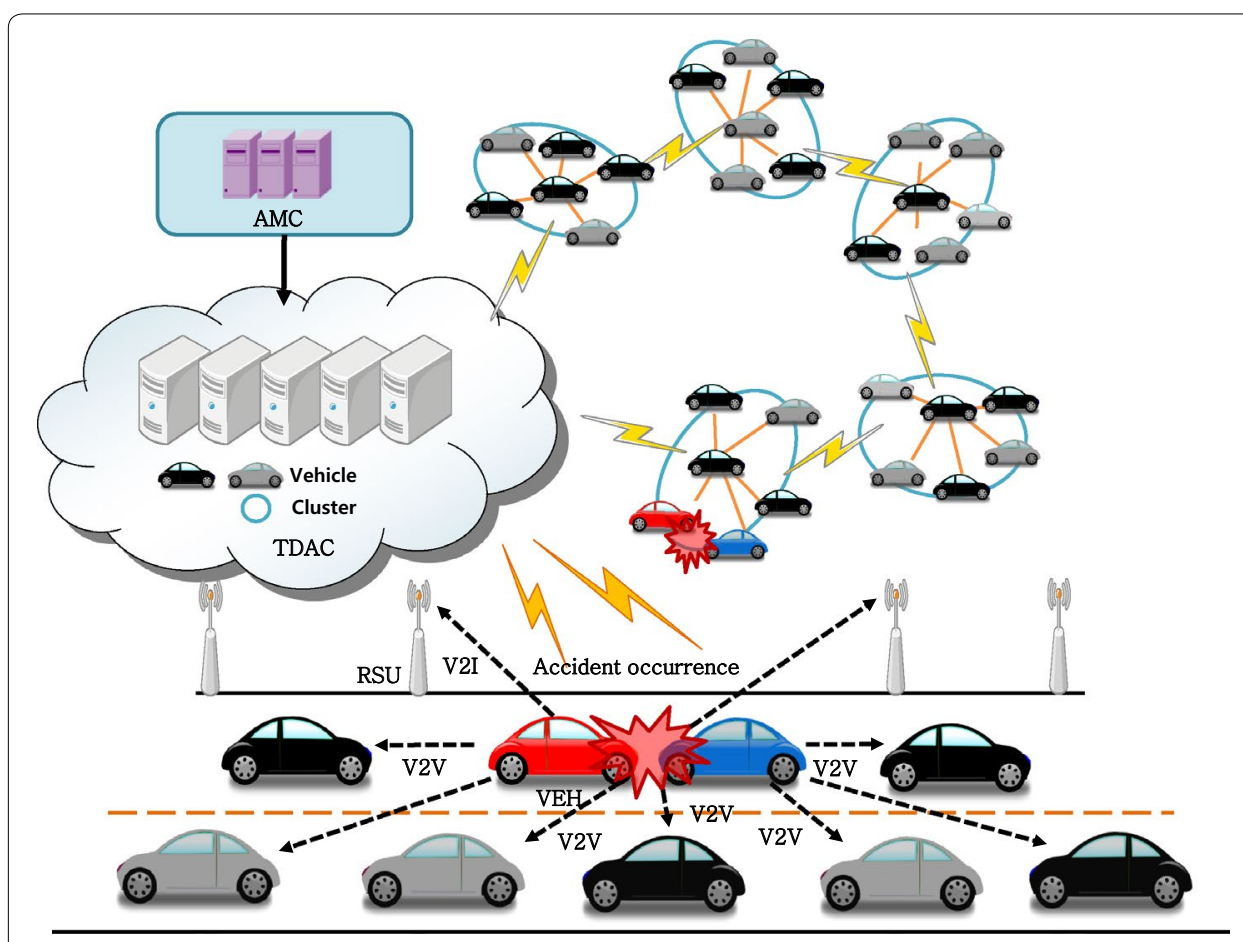

Fig. 3 The proposed Framework configuration 


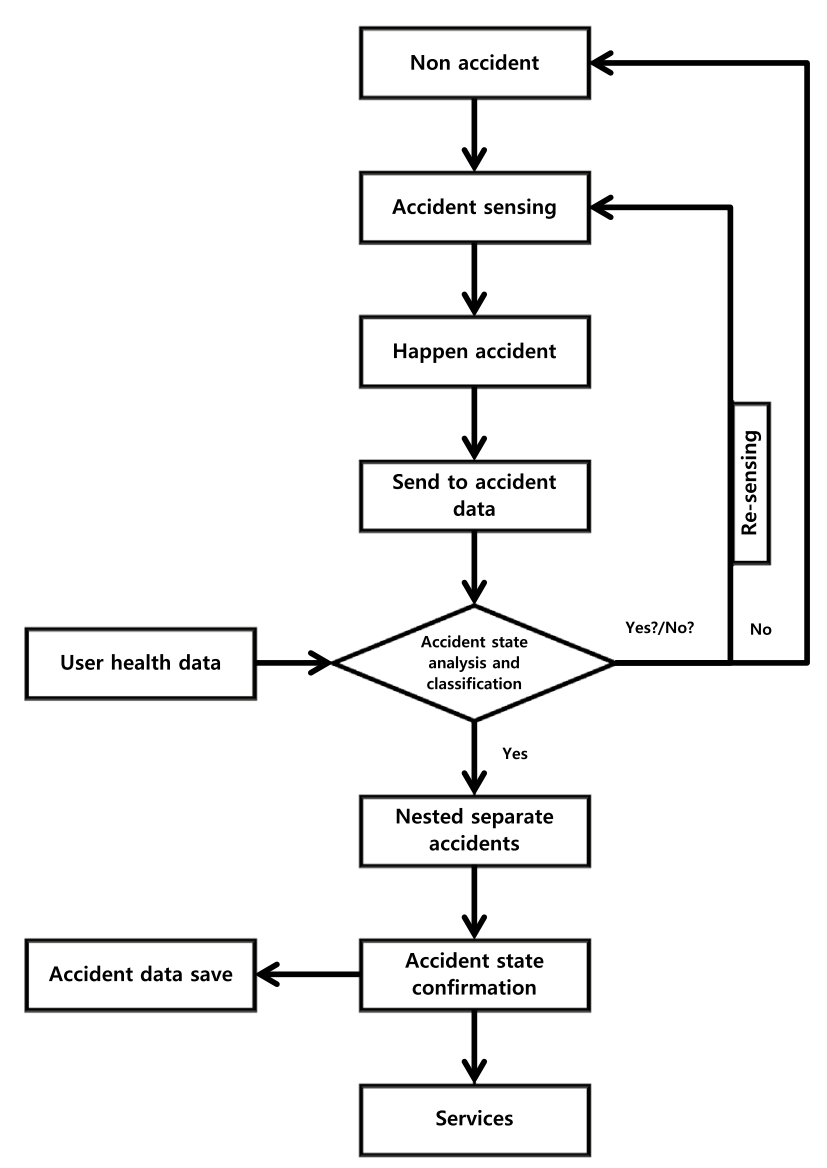

Fig. 4 Service of s-ITSF scenario

Table 2 Certain characteristic of study sites

\begin{tabular}{lllll}
\hline Characters & s-ITSF & [3] & [4] & [5] \\
\hline Active process & $\bigcirc$ & $\triangle$ & $\triangle$ & $\triangle$ \\
Traffic prevention/management processing & $\bigcirc$ & 0 & $\triangle$ & $\triangle$ \\
Data authentication & $\bigcirc$ & $\triangle$ & $\triangle$ & $\triangle$ \\
\hline
\end{tabular}

๑): Strong, O: Middle, $\triangle$ : Weak

for settlement of accident. The existing research manages an accident but does not manage it systematically manage the occurrence to settlement. On the other hand, the proposed s-ITSF provides an active accident management in overall steps from occurrence to settlement based on VCC. Traffic prevention/management processing, the existing research mainly focus on prevention of traffic jam and accident but do not provide a sufficient action after accident. s-ITSF is a system which prevents an accident by classifying and managing the whole situation of the traffic accident through TADC, AMC and AMO. Data authentication provides the authentication of the user's vehicle and confidentiality and integrity through encryption with TADC. The previous researches lack the security function for the health information shared since it provides only accident 
management service. On the other hand, s-ITSF may provide a higher reliable service through encryption of health information shared and authentication of user and vehicle.

\section{Conclusion}

ITS has been studied for long time but a fundamental solution has not been found due to lack of systematic accident management system in prevention and management of accident when a traffic accident takes place. s-ITSF proposed by this paper classifies the event before accident and after accident to provide an accident prevention service before accident and proposes an efficient and systematic SAM framework after accident by classifying the accident in hierarchy. The proposed framework provided a personalize service to identify the level of accident damage information to step through the AMC and AMO provided the personal services support to the accident area. In addition, it may provide the information to prevent an accident through collection and management of the accident information through TADC. s-ITSF is expected to provide an efficient and safe accident management preventing an accident being applied to the current ITS environment, provided that an additional research is needed in order to solve an overhead problem which may occur in big data processing.

\section{Authors' contributions}

JKL: main writing and also designing the total system and design of the total system; YSJ: research on related works, analyzing and improving the proposed system; JHP: total supervision of the paperwork, review, and comments, etc. All authors have read and approved the final manuscript.

\section{Author details}

${ }^{1}$ Department of Computer Science and Engineering, Seoul National University of Science and Technology, Seoul, South Korea. ${ }^{2}$ Department of Multimedia Engineering, Dongguk University, Seoul, South Korea.

\section{Acknowledgements}

This study was supported by the Research Program, which was funded by the Seoul National University of Science and Technology.

\section{Competing interests}

The authors declare that they have no competing interests.

Received: 12 October 2015 Accepted: 11 November 2015

Published online: 04 December 2015

\section{References}

1. Whaiduzzaman M, Sookhak M, Gani A, Buyya R (2014) A survey on vehicular cloud computing. J Netw Comput Appl 40:325-344

2. Lin X, Lu R, Zhang C, Zhu H, Ho PH, Xuemin (Sherman) S (2008) Security in vehicular ad hoc networks. Commun Mag IEEE 46(4):88-95

3. Zeadally S, Hunt R, Chen YS, Irwin A, Hassan A (2012) Vehicular ad hoc networks (VANETS): status, results, and challenges. Telecommun Sys 50(4):217-247

4. Vinh Hoa LA, Cavalli A (2014) Security attacks and solutions in vehicular ad hoc networks: a survey. Int J AdHoc Netwo Sys (IJANS) 4:1-20

5. Li C, Zhao C, Zhu L, Lin H, Li J (2014) Geographic routing protocol for vehicular ad hoc networks in city scenarios: a proposal and analysis. Int J Commun Syst 27:4126-4143

6. Mejri MN, Ben-Othman J (2014) Survey on VANET security challenges and possible cryptographic solutions. Veh Commun 1(2):53-66

7. Alvear D, Abreu O, Questa A, Alonso V (2013) Decision support system for emergency management: road tunnels. Tunn Undergr Space Technol 34:13-21

8. Ni D, Liu H, Ding W, Xie Y, Wang H, Pishro-Nik H, Yu Q (2012) Cyber-physical integration to connect vehicles for transformed transportation safty and efficiency. Adv Res Appl Artif Intell, Springer, 7345: pp 88-94

9. Wan J, Zhang D, Sun Y, Lin K, Zou C, Cai H (2014) VCMIA: a novel architecture for integrating vehicular cyber-physical systems and mobile cloud computing. Mob Netw Appl 19(2):153-160

10. Hussain R, Oh H (2014) Cooperation-aware VANET clouds: providing secure cloud services to vehicular ad hoc networks. J Info Process Sys 10(1):103-118

11. Wasef A, Shen X (2013) EMAP: Expedite Message Authentication Protocol for Vehicular Ad Hoc Networks". IEEE Trans Mob Comput 12(1):78-89 